\title{
Gut Microbiota Profile in Patients with Type 1 Diabetes Based on 16S rRNA Gene Sequencing: A Systematic Review
}

\author{
He Zhou $\mathbb{D}^{D}$, Xue Zhao, Lin Sun, Yujia Liu, You Lv, Xiaokun Gang, and Guixia Wang $\mathbb{D}$ \\ Department of Endocrinology and Metabolism, The First Hospital of Jilin University, Changchun 130021, China \\ Correspondence should be addressed to Guixia Wang; gwang168@jlu.edu.cn
}

Received 14 December 2019; Revised 7 August 2020; Accepted 20 August 2020; Published 28 August 2020

Academic Editor: Wen-Jun Tu

Copyright @ 2020 He Zhou et al. This is an open access article distributed under the Creative Commons Attribution License, which permits unrestricted use, distribution, and reproduction in any medium, provided the original work is properly cited.

\begin{abstract}
The gut microbiota has been presumed to have a role in the pathogenesis of type 1 diabetes (T1D). Significant changes in the microbial composition of T1D patients have been reported in several case-control studies. This study is aimed at systematically reviewing the existing literature, which has investigated the alterations of the intestinal microbiome in T1D patients compared with healthy controls (HCs) using $16 \mathrm{~S}$ ribosomal RNA-targeted sequencing. The databases of MEDLINE, EMBASE, Web of Science, and the Cochrane Library were searched until April 2019 for case-control studies comparing the composition of the intestinal microbiome in T1D patients and HCs based on 16S rRNA gene sequencing techniques. The Newcastle-Ottawa Scale was used to assess the methodological quality. Ten articles involving 260 patients with T1D and 276 HCs were included in this systematic review. The quality scores of all included studies were 6 -8 points. In summary, a decreased microbiota diversity and a significantly distinct pattern of clustering with regard to $\beta$-diversity were observed in T1D patients when compared with HCs. At the phylum level, T1D was characterised by a reduced ratio of Firmicutes/Bacteroidetes in the structure of the gut community, although no consistent conclusion was reached. At the genus or species level, T1D patients had a reduced abundance of Clostridium and Prevotella compared with HCs, whereas Bacteroides and Ruminococcus were found to be more enriched in T1D patients. This systematic review identified that there is a close association between the gut microbiota and development of T1D. Moreover, gut dysbiosis might be involved in the pathogenesis of T1D, although the causative role of gut microbiota remains to be established. Further well-controlled prospective studies are needed to better understand the role of the intestinal microbiome in the pathogenesis of T1D, which may help explore novel microbiota-based strategies to prevent and treat T1D.
\end{abstract}

\section{Introduction}

Type 1 diabetes (T1D) is a chronic autoimmune disease characterised by the immune-mediated destruction of insulinproducing pancreatic beta cells, usually occurring in children and young adults [1-3]. Although there is still some uncertainty about the aetiology of T1D, it is currently considered a multifactorial autoimmune disorder involving both genetic predisposition and environmental factors $[4,5]$. With the introduction of high-throughput sequencing, the structure of microflora can be analysed more comprehensively than before [6]. The intestinal microbiota, known as the "human second genome" [7], can coevolve with their host in a symbiotic relationship by combating pathogenic organisms [8], assisting in food digestion [9], maintaining the integrity of the intestinal epithelia [10], and promoting immunological development $[10,11]$. In the past decade, there has been growing evidence suggesting that gut dysbiosis may be a major contributor to T1D development [12]. A variety of studies have identified differences in the gut microbiota of healthy subjects and T1D patients [13]. In addition, growing evidence from well-controlled intervention studies in rodent models has supported the causative association between gut dysbiosis and T1D pathogenesis. The methods commonly used in these studies to alter the composition of gut microbiota include the use of probiotics, the use of antibiotics, fecal microbiota transplantation, and diet intervention. It has been proposed that the altered intestinal microbiota may impact 
T1D pathogenesis by increasing gut permeability [14], facilitating intestinal inflammation [15], and disturbing immunological maturation $[16,17]$. Nevertheless, given the paucity of well-controlled studies in humans owing to the lack of corrective methods for confounding factors, gut microbiota as a causal factor leading to the progression of T1D remains speculative. Once the causative relationship between gut microbiota and T1D development is confirmed and the related pathophysiological mechanisms are delineated, the gut microbiota will be a novel area to explore for new preventative or therapeutic strategies for T1D. The discovery of a clear association between gut dysbiosis and T1D is of significant clinical importance as microbiota-based interventions such as probiotics can reduce or even prevent the burdensome requirement of injected insulin.

The $16 \mathrm{~S}$ ribosomal RNA (rRNA) gene sequencing technique has become the most widely used method to investigate the composition of microbial ecosystems in recent years [18, 19]. Compared with traditional culture-based methods and previously used low-resolution methods, which can only identify specific bacteria, 16S rRNA-based sequencing as a rapid, cost-effective, and less labour-intensive microbial detection approach can analyse the composition of the whole microbial community and significantly improve the resolution of bacterial identification [6]. To date, a variety of case-control studies have observed perturbed intestinal microflora in T1D patients compared with healthy controls (HCs) using 16S rRNA gene sequencing. Given that different bacterial detection technologies have distinct levels of discrimination, which limits the ability to make accurate and specific comparisons across studies [20], we performed a systematic review to provide an overview of the intestinal microflora profile in patients with T1D based on 16S rRNA gene sequencing.

\section{Methods}

The systematic review was performed according to the PRISMA guidelines (Preferred Reporting Items for Systematic Reviews and Meta-Analyses), and the protocol was registered at PROSPERO (registration number: CRD42019137493).

2.1. Literature Search. To identify relevant studies on the intestinal microbial profile associated with T1D, an electronic search was conducted using MEDLINE, EMBASE, Web of Science, and the Cochrane Library databases from inception until April 2019. This search strategy used a combination of $\mathrm{MeSH}$ terms and keywords pertaining to gut microbiota and T1D. The following search terms were employed: ("Microbiota" OR "Microbiome" OR "Microflora" OR "Microbes" OR "Microbial Community" OR "Microbiota Composition" OR "Flora” OR “Dysbiosis" OR "Bacteria”) AND (“Type 1 Diabetes" OR "Type 1 Diabetes Mellitus" OR “Autoimmune Diabetes" OR "InsulinDependent Diabetes Mellitus" OR “T1DM" OR "Beta-Cell autoimmunity"). The reference lists of the selected studies were manually searched for additional relevant trials.

2.2. Eligibility Criteria. The inclusion criteria were as follows: (1) case-control studies comparing gut microbiota in patients with diagnosed T1D and HCs, (2) microbiota analysis using $16 \mathrm{~S}$ rRNA gene sequencing, and (3) studies published in English.

The exclusion criteria were as follows: (1) studies without HCs; (2) cases being prediabetic subjects with islet autoimmunity, not diagnosed with T1D; (3) microbiota analysis using other microbial detection methods; (4) animal studies; and (5) non-English language studies.

2.3. Data Extraction. The following data were extracted from eligible articles by two independent reviewers: (1) first author; (2) year of publication; (3) country of origin; (4) characteristics of cases and HCs (including sample size, mean age, and sex ratio); (5) sample source; (6) DNA extraction method, sequenced region, sequencing platform, analysis platform, and referred database used in these studies; (6) indices of microbial diversity; and (7) major findings of intestinal microbiome in patients with T1D compared to those in HCs. Discrepancies between the two reviewers were resolved by consulting a third reviewer.

2.4. Quality Assessment. The Newcastle-Ottawa Scale (NOS) was used to assess the quality of included studies in this systematic review. The NOS is considered an effective way to evaluate the quality of case-control studies, and a total of nine items are included in NOS. The selection criteria contain four items: (1) the adequate case definition, (2) representativeness of the cases, (3) control selection, and (4) control definition. The comparability criteria include comparability of cases and controls according to the design or analysis. The exposure criteria contain three aspects: (1) ascertainment of exposure, (2) the same method of ascertainment for cases and controls, and (3) nonresponse rate.

\section{Results}

3.1. Study Selection. Following the search strategy, a total of 1356 articles were initially obtained including 469 articles in MEDLINE, 631 in EMBASE, 153 in Web of Science, and 103 in the Cochrane Library. The manual search of reference lists of relevant studies yielded no additional studies. A total of 585 articles were duplicates and were removed. The remaining 771 studies were screened based on the titles and abstracts, of which 731 articles were discarded mainly because of incorrect article types, including editorials, letters, comments, case reports, and reviews as well as incorrect topic and animal studies, leading to 40 articles remaining for fulltext review. After reviewing the full text, 30 studies were excluded for the following reasons: (1) 13 studies used other microbial detection methods rather than 16S rRNA gene sequencing; (2) cases of 11 studies were prediabetic subjects with islet autoimmunity, not established T1D patients; (3) 3 studies did not contain a healthy control group; (4) 2 studies provided irrelevant outcomes; and (5) 1 study was not written in English. Finally, a total of 10 studies met the inclusion criteria and were included in this systematic review (Figure 1).

3.2. Study Characteristics. The 10 selected articles included a total of 536 individuals with 260 cases of T1D and 276 HCs. 

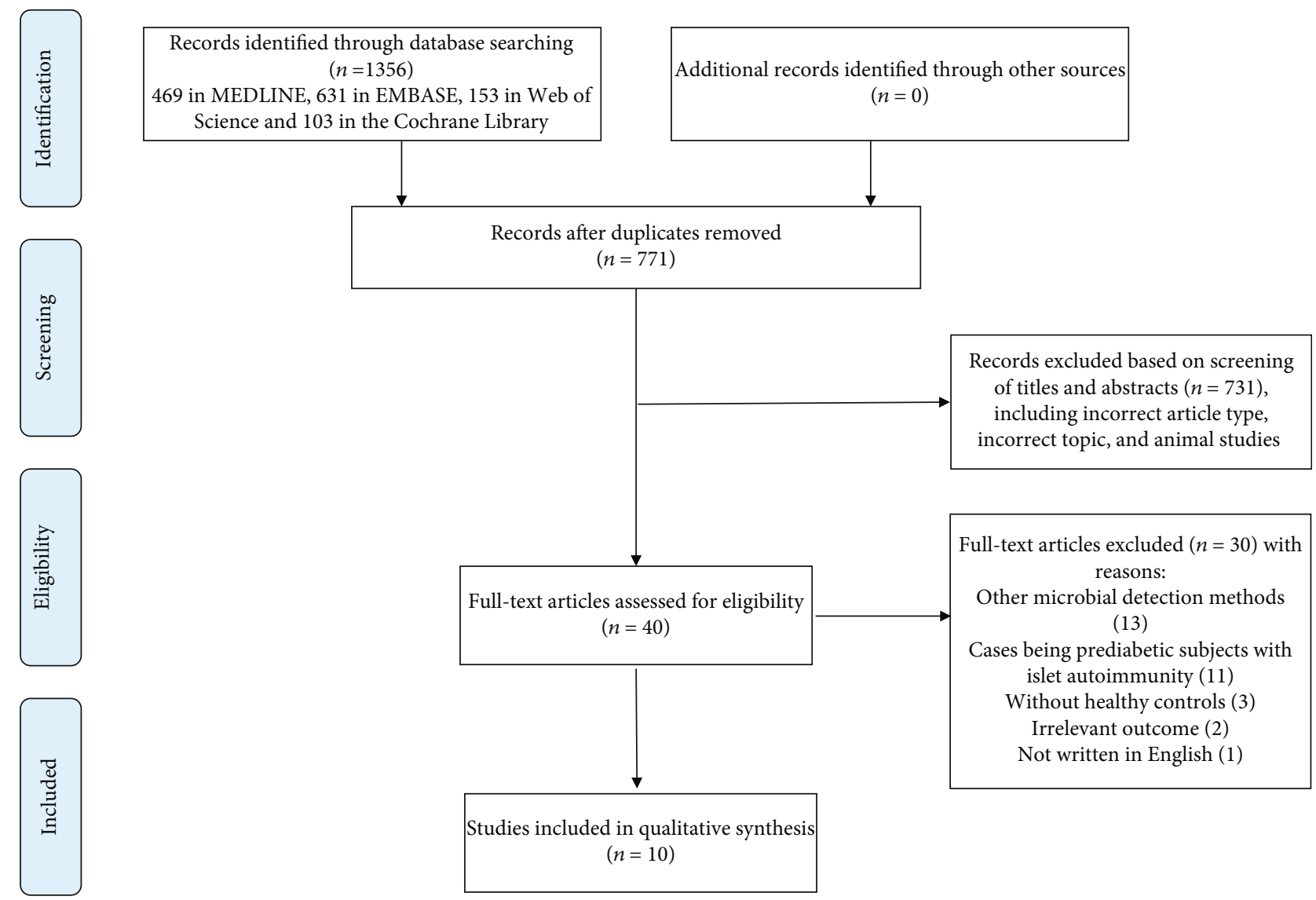

Studies included in qualitative synthesis $(n=10)$

FIgURE 1: Flowchart of the selected studies.

The general characteristics and main findings of the included articles are summarised in Table 1 . The majority of the eligible studies were published within the last three years. Twelve countries were included in the included studies: Azerbaijan, Jordan, Nigeria, Sudan, Brazil, China, Spain, Poland, the Netherlands, Italy, the UK, and Mexico. The mean age of the patients with T1D ranged from 11 to 36 years and 11 to 38 years in HCs. The proportion of male participants varied from $27.3 \%$ to $100 \%$ in patients with T1D and from $30.4 \%$ to $100 \%$ in HCs; additionally, one study was excluded from the gender calculation because its gender description was not available. Except for one study using mucosal biopsies, other studies analysed fecal samples to assess shifts in the gut microbiota composition. The QIAamp DNA Stool Mini Kit was most extensively used for DNA extraction. Different 16S rRNA variable regions were targeted for DNA amplification: region V1-V2 (1 study), region V2-V3 (1 study), region V3-V4 (4 studies), region V3-V5 (1 study), and region V4 (3 studies). Seven studies chose the Illumina MiSeq platform as the sequencing platform, and three studies were performed on the 454 platform. Qiime and Mothur were most widely adopted with respect to data analysis platforms. Although two studies did not report which database was referred, the remaining studies used the Silva database, the Greengenes database, and the Ribosomal Database Project for mapping the sequences. The most frequently utilised estimator for $\alpha$ - diversity was the Chaol index, followed by the Shannon index, although one study did not provide information on which index was employed. The alterations of gut microbiota mainly focused on the dominant phylum and a variety of different specific bacterial genera or subspecies, that is, Clostridium, Bacteroides, Prevotella, Bifidobacterium, Ruminococcus, and Streptococcus. The quality scores of the eligible studies assessed by NOS are shown in Table 2, and all included studies showed medium (6-7 points) to high (8 points) quality.

3.3. Microbiota Diversity in Patients with T1D. The richness, evenness, and $\alpha$-diversity can all be used to express the diversity of species. All included studies in this systematic review have investigated the bacterial diversity in T1D patients compared with HCs, although different indexes were utilised. One study conducted by Leiva-Gea et al. found that one of the evidential gut microbiome signatures of patients with T1D was the decreased $\alpha$-diversity estimated by the Shannon index in comparison to HCs [24]. Another study by Qi et al. evaluating the microbial diversity using the Shannon and Chao indices showed that there was a reduced microbiota richness when evaluated using the Chao index in T1D patients compared with that in HCs [29]. A $\beta$-diversity represents the dissimilarity between the two gut communities. A total of six studies in this review have analysed the $\beta$-diversity of gut microflora, and four of them consistently 


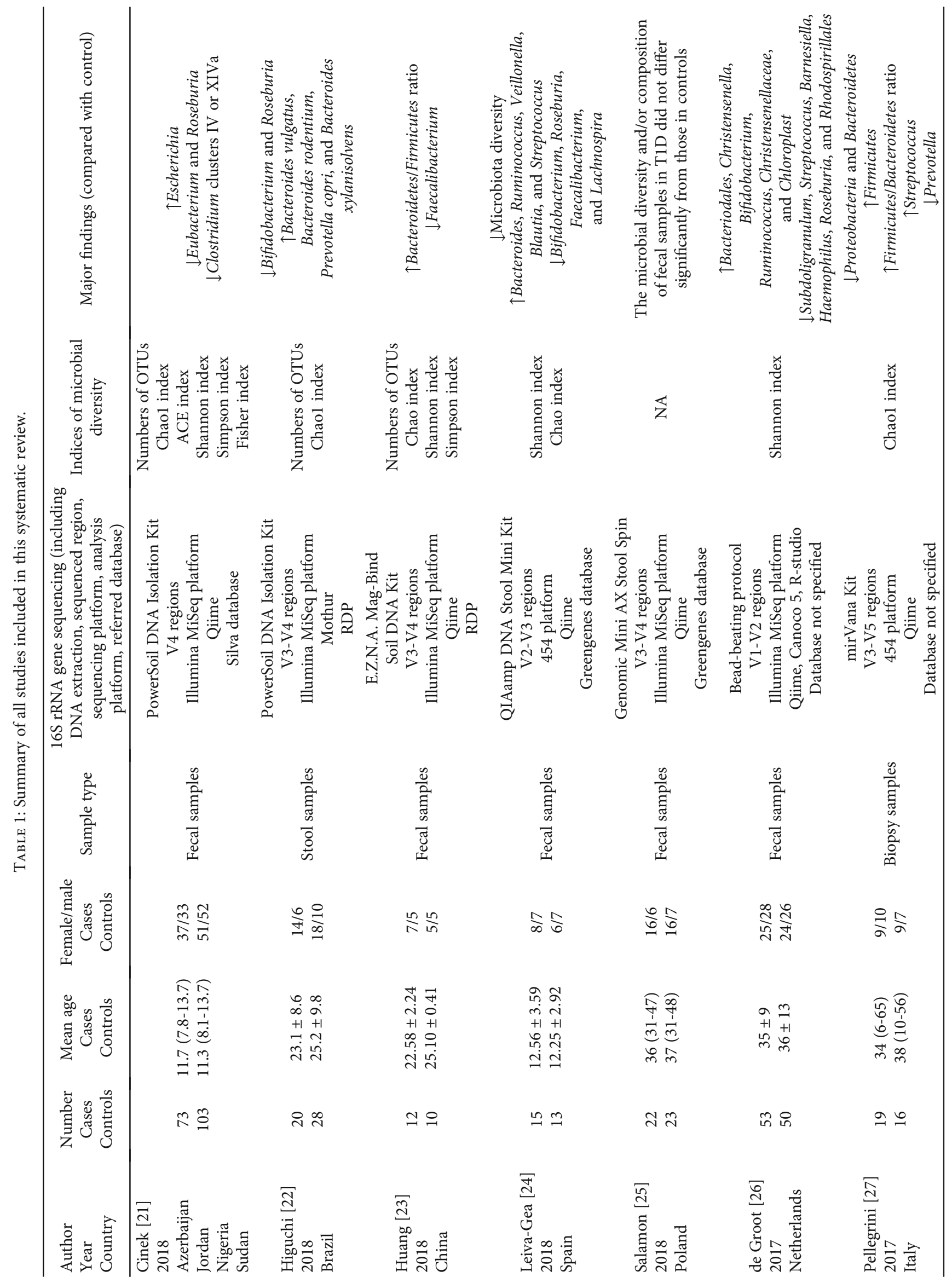




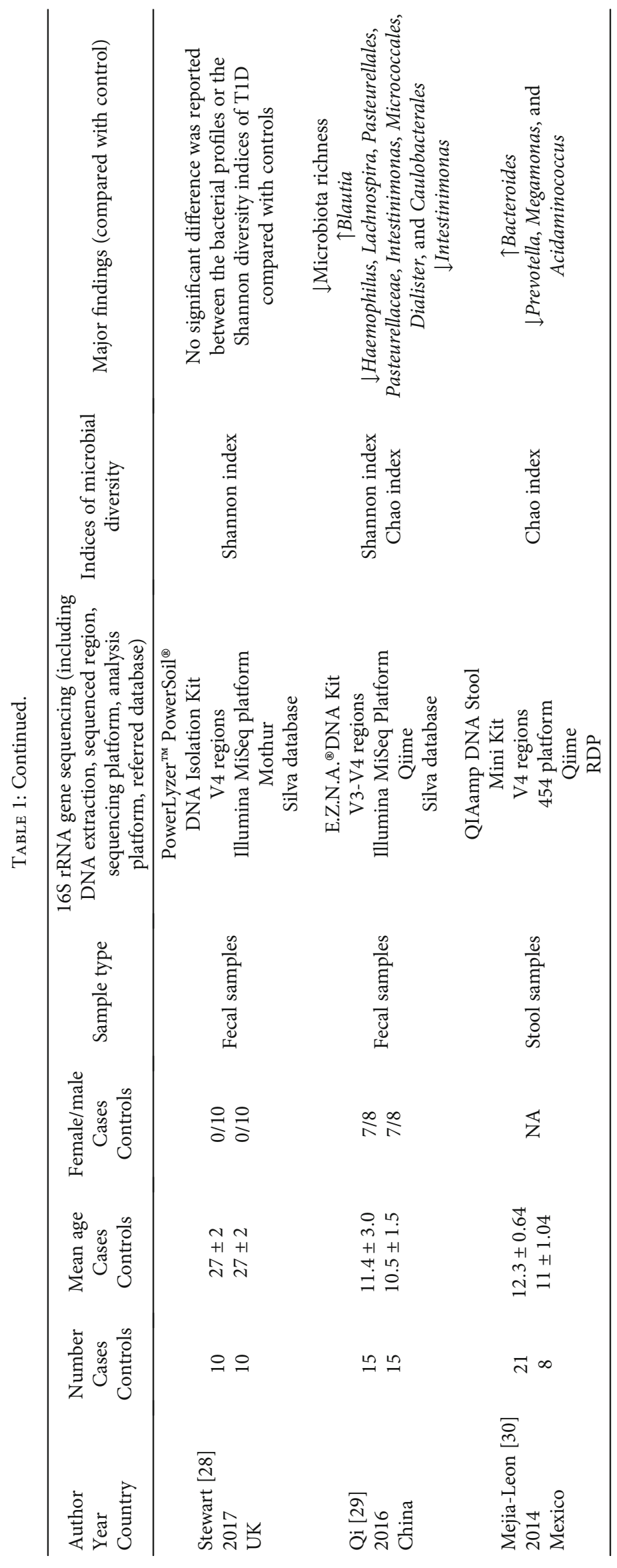


TABLE 2: Quality assessment of included studies.

\begin{tabular}{lccccc}
\hline Author & Year & Selection & Comparability & Exposure & $\begin{array}{c}\text { Total } \\
\text { score }\end{array}$ \\
\hline Cinek & 2018 & 4 & 1 & 2 & 7 \\
Higuchi & 2018 & 3 & 1 & 2 & 6 \\
Huang & 2018 & 4 & 1 & 2 & 7 \\
Leiva-Gea & 2018 & 4 & 2 & 2 & 8 \\
Salamon & 2018 & 3 & 1 & 2 & 6 \\
de Groot & 2017 & 3 & 1 & 2 & 6 \\
Pellegrini & 2017 & 4 & 2 & 2 & 8 \\
Stewart & 2017 & 3 & 1 & 2 & 6 \\
Qi & 2016 & 4 & 2 & 2 & 8 \\
Mejia- & 2014 & 3 & 2 & 2 & 7 \\
Leon & & & & &
\end{tabular}

identified that the gut microbiome of T1D patients showed a remarkably distinct pattern of clustering when compared with that of HCs $[22,24,25,30]$.

3.4. Altered Composition of Gut Microbiota in Patients with T1D. The majority of the studies included in the present systematic review have identified the major taxonomic alterations of microbiota structure, especially at the genus or species level. Only two studies identified that the microbial profile in T1D patients did not differ significantly from that in HCs $[25,28]$. This may be explained by differences in dietary habits, geographical environments, and sample sources across studies.

A notable alteration of gut bacterial community at the phyla level was the reduction in the Firmicutes/Bacteroidetes (F/B) ratio in T1D patients compared with HCs, which has been observed in two studies [23, 24]. Two other studies, whose major taxa explained the altered microbiota profile, were reflected at the genus level or species level; both reported that the $\mathrm{F} / \mathrm{B}$ ratio was not significantly different between the T1D patients and HCs. However, these two studies found some genus or species belonging to the Firmicutes, such as Lachnospira, Dialister, Intestinimonas, and Caulobacterales were significantly reduced, while those belonging to Bacteroidetes, such as Bacteroides, were increased $[29,30]$. These results suggested that the alterations of Firmicutes and Bacteroidetes might be involved in T1D development. Nevertheless, conflicting results have also been published. A study evaluating the microbial composition in duodenal biopsies rather than stool samples found that the ratio of F/B was markedly elevated in T1D patients when compared with HCs [27].

At the genus or species level, six studies consistently demonstrated a significant decline in the abundance of butyrateproducing species primarily within Clostridium in patients with T1D when compared with corresponding HCs [21-24, $26,29]$. In these studies, the most commonly detected butyrate-producing species are Roseburia faecis (a member of Clostridium cluster XIVa) and Faecalibacterium prausnitzii (a member of Clostridium cluster IV). Moreover, Intestinimonas, a newly isolated butyrate-producing bacterium, was also found to be significantly lower in T1D patients in a study performed by Qi et al. [29]. The relative abundance of Prevotella, a mucin-degrading bacterium and a marker of elevated mucin synthesis, was also significantly lower in T1D patients than in HCs $[27,30]$. In contrast, three studies consistently revealed that Bacteroides positively correlated with the development of T1D [24, 26, 30]. Several species belonging to Bacteroides, including Bacteroides vulgatus, Bacteroides rodentium, and Bacteroides xylanisolvens, were also significantly higher in T1D patients than in HCs [22]. Similarly, the abundance of Ruminococcus also showed an increasing trend in T1D patients [24, 26]. However, inconsistent results were also reported in this systematic review. Bifidobacterium was found to be significantly lower in T1D patients than in HCs in two studies $[22,24]$; however, it was reported to be higher in another study [26]. Two studies found that Streptococcus was more abundant in T1D patients than in HCs [24, 27], whereas another study reported contrasting results [26].

3.5. Inflammatory Status along with Gut Dysbiosis in T1D. Eligible studies also determined the T1D-specific inflammatory profile along with the altered composition of gut microbiota in T1D patients. Higuchi et al. demonstrated that the levels of inflammatory interleukin-6 (IL-6) were significantly higher in the plasma of T1D patients than in that of HCs. Additionally, the change in IL-6 plasma concentration correlated with the relative abundance of Ruminococcaceae and Ruminococcus members [22]. The elevated levels of IL-6 in T1D were reinforced by another study in this review, in which the authors identified increased levels of proinflammatory cytokines including IL- $1 \beta$, IL- 6 , and TNF- $\alpha$ and decreased levels of anti-inflammatory cytokines including IL-10 and IL-13, which significantly correlated with different bacterial groups in T1D patients [24]. They also found a dysbiosis-associated increase in zonulin levels, which indicates an increase in the gut permeability [24]. Pellegrini et al. observed an increased expression of genes specific for T1D inflammation in duodenal mucosa biopsies of T1D patients; it was linked to the altered relative abundance of specific gut microbiota [27]. Furthermore, immunohistochemical analysis of the duodenal mucosa confirmed the inflammatory status with a greater monocyte/macrophage lineage infiltration in the tissues of T1D patients than in those of HCs [27].

\section{Discussion}

Both experimental and observational studies have focused on the alterations of the intestinal microbiome in T1D patients because identifying microbial signatures is a critical step towards providing new insight into the diagnostic or therapeutic strategies for T1D. In the present study, considering that the method of bacterial analysis might be an affecting factor for microbial identification, we explored the shifts of the intestinal microbiome in T1D patients based on 16S rRNA-targeted sequencing to minimise the methodology-based heterogeneity. To our knowledge, our study is the first systematic review exploring the 
relationships between microbial alterations and the development of T1D based on $16 \mathrm{~S}$ rRNA-targeted sequencing around the world.

Data from eligible studies concordantly determined a reduced microbiota diversity as well as a significantly distinct pattern of microbiota clustering in T1D patients when compared with HCs. Reduced gut microbial diversity has been previously reported in prediabetic subjects with T1D-associated autoantibodies when compared with autoantibody-negative subjects [31-33]. It has also been detected prior to the appearance of autoantibodies in children at risk for T1D [34], indicating that decreased microbial diversity might be involved in the autoimmune process. In addition, the diversity of microbiota is inversely correlated with several immunerelated disorders such as atopic eczema [35], inflammatory bowel disease [36], chronic urticaria [37], and allergic asthma [38]. Microbial diversity is a crucial property of a healthy gastrointestinal ecosystem [39]. According to the hygiene hypothesis, advances in medicine and improved sanitation have changed the microbial environment exposure of humans, characterised by a lack of microbial stimulation and reduced microbial diversity in early childhood, eventually leading to an increase in the incidence of allergy and immune-related disorders [40, 41]. However, the exact mechanism of reduced diversity related to the pathogenesis of T1D is not clear and needs more attention in future studies.

At the phylum level, a decreased $\mathrm{F} / \mathrm{B}$ ratio in the structure of the gut community in T1D cases was observed because of reduced levels of Firmicutes and/or increased levels of Bacteroidetes. However, consistent conclusions have not been reached owing to the divergence in the results. A lower F/B ratio is consistent in patients with T1D-associated autoimmunity from cohort studies, in which the stool samples were collected prospectively before the onset of T1D among children genetically at risk for this disorder. It was also reported that the ratio of F/B decreased over time in autoantibodypositive children $[31,32]$. A study using polymerase chain reaction- (PCR-) denaturing gradient gel electrophoresis and real-time quantitative PCR (RT-qPCR) to detect the gut microbiota also reported a lower $\mathrm{F} / \mathrm{B}$ ratio in patients with T1D than in HCs [42]. However, despite these findings, the association of such changes in the F/B ratio with T1D has not yet been elucidated. Future studies should confirm whether the decreased F/B ratio is a microbial signature of the gut community of patients with T1D and elucidate its association with T1D.

At the genus or species level, there were consistent changes in the microbial composition in T1D patients compared with HCs. A reduced abundance of Clostridium species, especially clusters IV and XIVa, was found in T1D patients. It was found that these microorganisms perform physiological functions by producing butyrate [43]. Commensal microbes in the intestine can ferment dietary fibres and produce short-chain fatty acids (SCFAs) such as acetate, propionate, and butyrate [44]. Butyrate plays a pivotal role in inducing $\mathrm{T}$ regulatory (Treg) cell differentiation in the gut mucosa, which could inhibit the immune response by secreting cytokines such as IL-10 $[45,46]$. In addition to its antiinflammatory activity, butyrate has also been recognised for its ability to enhance gut integrity by increasing tight junction (TJ) production [47] as well as facilitating mucin synthesis [48]. A potential pathway by which intestinal microbes influence the tightness of a TJ seems to be through the rise in zonulin concentration, which is a novel indicator of intestinal permeability $[49,50]$. Faecalibacterium has been regarded as a next generation probiotic owing to its several healthpromoting and anti-inflammatory properties [51]. As noted above, there was a specific inflammatory profile along with the altered composition of gut microbiota in T1D patients $[22,24,27]$. The reduction of butyrate-producing species might contribute to T1D progression by increasing the gut permeability and inducing chronic low-grade inflammation, subsequently eliciting a systemic immune response through a greater exposure to bacterial antigens $[49,52,53]$. Prevotella, a mucin-degrading bacterium, was found to be lower in T1D individuals than in HCs. In line with our findings, the numbers of Prevotella and Akkermansia (another mucin-degrading bacteria) were also reduced in autoantibody-positive subjects compared with matched autoantibody-negative controls [32]. The decline of mucusdegrading microbes may be correlated with the decreased biosynthesis of mucus during T1D development. In our systematic review, the relative abundance of Bacteroides consistently showed a higher level in patients with T1D. Similar findings were described in antibody-positive individuals when compared with antibody-negative subjects [32, 33]. Bacteroides are acetate- and propionate-producing bacteria [54], and their by-products cannot increase mucin production like butyrate [55]. It has been suggested that these bacteria might contribute to $\mathrm{T} 1 \mathrm{D}$ development by thinning the mucus layer, increasing the gut permeability, and leading to chronic inflammation, in which case luminal antigens will escape from the gut and eventually promote islet-directed autoimmune responses $[32,56]$. In addition, a recent study identified that gut-derived bacterial lipopolysaccharides from Bacteroides may suppress the innate immune signalling and endotoxin tolerance. Thus, this precludes the education and maturation of the immune system in early life [57]. As mentioned earlier, Ruminococcus also exhibited elevated levels in T1D patients. It has been established that Ruminococcus has proinflammatory effects and is positively correlated with irritable bowel syndrome [58]. However, the role of Ruminococcus in T1D development has not been well elucidated. Several studies found a reduced number of Bifidobacterium and an increased number of Streptococcus in T1D patients, whereas no consistent conclusions have been reached. Members of the genus Bifidobacterium are believed to confer positive health benefits to their host, and several Bifidobacterium taxa have been commonly used as probiotics [59]. There are several mechanisms that explain the inverse correlation between Bifidobacterium and T1D development: Bifidobacterium can produce lactate, which may be transformed into net butyrate [60], thus having the capacity to protect intestinal permeability [61]. Also, Bifidobacterium has been documented to promote the generation and function of Treg cells [62] and their subset type 1 regulatory $\mathrm{T}(\operatorname{Tr} 1)$ cells, which can also produce IL-10 [63], thereby preventing intestinal inflammation. While the influence of Streptococcus on the development of 
T1D remains unclear, it was reported to be increased in patients with multiple sclerosis (MS). It was also speculated that Streptococcus was involved in the pathogenesis of MS by inducing the differentiation of Th17 cells [64]. Thus, the inconsistency in the numbers of Bifidobacterium and Streptococcus in T1D patients needs further exploration and confirmation.

Currently, the pancreatic islet autoantibodies are considered the most reliable biomarkers for T1D in both children and adults [65]. The distinct gut microbiota profile in T1D patients may serve as an alternative biomarker for T1D; moreover, stool sampling is easy to perform and noninvasive. Furthermore, the accumulated data suggest that over time, the trajectory of gut microbiota develops in a different way in children with T1D and in those without T1D before the onset of the disease. Thus, the dysbiosis of the gut microbiota could also be potentially used as a promising biomarker for the early diagnosis of T1D in genetically susceptible children. The intestinal microbiota is also a potential preventative and therapeutic target for T1D. Specific bacterial genera such as Bifidobacterium and Lactobacillus as well as the metabolites produced by gut microbiota, such as SCFAs, can be used as probiotics and prebiotics, respectively, owing to their favourable impact on the gut environment. Thus far, several animal studies have reported the protective effect of probiotics and prebiotics on T1D [66-68]. Recently, a randomised, placebo-controlled trial in children with T1D demonstrated that the administration of prebiotics for at least one year could increase the relative abundance of Bifidobacterium and improve the beta cell function [69]. However, such randomised controlled studies are still limited, and further largescale clinical trials are warranted.

There are a few limitations to our study. First, a major limitation in all studies is the inherent nature of the crosssectional design, which cannot provide causal relationships, but only an association between the gut microbiota and T1D development. Second, another limiting factor is the heterogeneity between study populations. The studies included in this review were performed across different countries; thus, the dietary habits might vary considerably. Geographical regions and the diet are major factors that can exert a great impact on the gut community, making it difficult to draw a consistent microbiota profile in T1D patients and even in HCs. Third, the sample type was also a source of heterogeneity. In our systematic review, the majority of the studies used fecal samples, and only one study examined the mucosa biopsy of the intestine. It is recognised that the bacterial composition in feces and biopsy samples may differ in the same individual. Additionally, given that the mucosaepithelia associated microbiota is likely to have a more intimate interplay with the intestinal epithelium and immune cells, the analysis of microflora using biopsies may be more appropriate [70]. Given the heterogeneity of the study characteristics and results, we conducted a narrative systematic review rather than a meta-analysis.

\section{Conclusions}

This review systematically assessed studies determining the alterations of gut microbiota composition based on $16 \mathrm{~S}$
rRNA-targeted sequencing in T1D patients compared with that in HCs. In summary, the data from included studies support a relationship between microbiota abnormalities and T1D development. A reduced microbiota diversity and a significantly distinct pattern of clustering with regard to $\beta$ diversity were observed in T1D patients when compared with those in HCs. At the genus level, T1D was characterised by a reduced $\mathrm{F} / \mathrm{B}$ ratio in the structure of the gut community, although no consistent conclusion was reached. At the genus or species level, T1D patients consistently showed a reduced abundance of Clostridium and Prevotella when compared with HCs, whereas Bacteroides and Ruminococcus were found to be more enriched in T1D patients. However, there is not enough consistency in the alteration of Bifidobacterium and Streptococcus, which needs to be further confirmed in the future. It seems that the gut microbiota profile in T1D patients was associated with impaired epithelial integrity, low-grade inflammation, and autoimmune response, implying that alterations in these bacterial populations might contribute to the pathogenesis of $\mathrm{T} 1 \mathrm{D}$, although the causative role of dysbiosis remains to be established. Further wellcontrolled prospective studies are warranted to better understand the role of the gut microbiota in the development of T1D, which may help explore new microbiota-based strategies to prevent and treat T1D.

\section{Data Availability}

The data used to support the findings of this study are available from the corresponding author upon request.

\section{Conflicts of Interest}

The authors declare that there is no conflict of interest regarding the publication of this paper.

\section{Acknowledgments}

This work was supported by grants from the Key Laboratory of Endocrine and Metabolic Diseases of Jilin Province (20190901006JC) and the Science and Technology Department Open Project (20170623092TC-01 and 20180623083TC-01).

\section{References}

[1] E. Gulden, F. S. Wong, and L. Wen, "The gut microbiota and type 1 diabetes," Clinical Immunology, vol. 159, no. 2, pp. 143-153, 2015.

[2] M. A. Atkinson, G. S. Eisenbarth, and A. W. Michels, “Type 1 diabetes," The Lancet, vol. 383, no. 9911, pp. 69-82, 2014.

[3] A. M. Abdellatif and N. E. Sarvetnick, "Current understanding of the role of gut dysbiosis in type 1 diabetes," Journal of Diabetes, vol. 11, no. 8, pp. 632-644, 2019.

[4] F. Pociot and M. F. McDermott, "Genetics of type 1 diabetes mellitus," Genes and Immunity, vol. 3, no. 5, pp. 235-249, 2002.

[5] S. Esposito, G. Toni, G. Tascini, E. Santi, M. G. Berioli, and N. Principi, "Environmental factors associated with type 1 diabetes," Frontiers in Endocrinology, vol. 10, p. 592, 2019. 
[6] B. M. Forde and P. W. O'Toole, "Next-generation sequencing technologies and their impact on microbial genomics," Briefings in Functional Genomics, vol. 12, no. 5, pp. 440-453, 2013.

[7] J. Qin, MetaHIT Consortium, R. Li et al., "A human gut microbial gene catalogue established by metagenomic sequencing," Nature, vol. 464, no. 7285, pp. 59-65, 2010.

[8] B. Stecher and W. D. Hardt, "Mechanisms controlling pathogen colonization of the gut," Current Opinion in Microbiology, vol. 14, no. 1, pp. 82-91, 2011.

[9] A. B. Shreiner, J. Y. Kao, and V. B. Young, "The gut microbiome in health and in disease," Current Opinion in Gastroenterology, vol. 31, no. 1, pp. 69-75, 2015.

[10] J. M. M. Natividad, V. Petit, X. Huang et al., "Commensal and probiotic bacteria influence intestinal barrier function and susceptibility to colitis in $\mathrm{Nod}_{1}{ }^{-/-}$; Nod2 $2^{-/-}$mice," Inflammatory Bowel Diseases, vol. 18, no. 8, pp. 1434-1446, 2012.

[11] M. Dave, P. D. Higgins, S. Middha, and K. P. Rioux, "The human gut microbiome: current knowledge, challenges, and future directions," Translational Research, vol. 160, no. 4, pp. 246-257, 2012.

[12] O. Vaarala, "Human intestinal microbiota and type 1 diabetes," Current Diabetes Reports, vol. 13, no. 5, pp. 601-607, 2013.

[13] S. Bibbò, M. P. Dore, G. M. Pes, G. Delitala, and A. P. Delitala, "Is there a role for gut microbiota in type 1 diabetes pathogenesis?," Annals of Medicine, vol. 49, no. 1, pp. 11-22, 2017.

[14] E. Bosi, L. Molteni, M. G. Radaelli et al., "Increased intestinal permeability precedes clinical onset of type 1 diabetes," Diabetologia, vol. 49, no. 12, pp. 2824-2827, 2006.

[15] M. Westerholm-Ormio, O. Vaarala, P. Pihkala, J. Ilonen, and E. Savilahti, "Immunologic activity in the small intestinal mucosa of pediatric patients with type 1 diabetes," Diabetes, vol. 52, no. 9, pp. 2287-2295, 2003.

[16] K. M. Bendtsen, L. Fisker, A. K. Hansen, C. H. F. Hansen, and D. S. Nielsen, "The influence of the young microbiome on inflammatory diseases-lessons from animal studies," Birth Defects Research Part C: Embryo Today: Reviews, vol. 105, no. 4, pp. 278-295, 2015.

[17] E. M. Brown, D. J. Kenny, and R. J. Xavier, "Gut microbiota regulation of T cells during inflammation and autoimmunity," Annual Review of Immunology, vol. 37, no. 1, pp. 599-624, 2019.

[18] F. J. Whelan and M. G. Surette, "A comprehensive evaluation of the sllp pipeline for 16S rRNA gene sequencing analysis," Microbiome, vol. 5, no. 1, p. 100, 2017.

[19] L. Mancabelli, C. Milani, G. A. Lugli et al., "Identification of universal gut microbial biomarkers of common human intestinal diseases by meta-analysis.," FEMS Microbiology Ecology, vol. 93, no. 12, 2017.

[20] E. K. Wright, M. A. Kamm, S. M. Teo, M. Inouye, J. Wagner, and C. D. Kirkwood, "Recent advances in characterizing the gastrointestinal microbiome in Crohn's disease: a systematic review," Inflammatory Bowel Diseases, vol. 21, no. 6, pp. 1219-1228, 2015.

[21] O. Cinek, L. Kramna, K. Mazankova et al., "The bacteriome at the onset of type 1 diabetes: a study from four geographically distant African and Asian countries," Diabetes Research and Clinical Practice, vol. 144, pp. 51-62, 2018.

[22] B. S. Higuchi, N. Rodrigues, M. I. Gonzaga et al., "Intestinal dysbiosis in autoimmune diabetes is correlated with poor glycemic control and increased interleukin-6: a pilot study," Frontiers in Immunology, vol. 9, 2018.
[23] Y. Huang, S. C. Li, J. Hu et al., "Gut microbiota profiling in Han Chinese with type 1 diabetes," Diabetes Research and Clinical Practice, vol. 141, pp. 256-263, 2018.

[24] I. Leiva-Gea, L. Sánchez-Alcoholado, B. Martín-Tejedor et al., "Gut microbiota differs in composition and functionality between children with type 1 diabetes and MODY2 and healthy control subjects: a case-control study," Diabetes Care, vol. 41, no. 11, pp. 2385-2395, 2018.

[25] D. Salamon, A. Sroka-Oleksiak, P. Kapusta et al., "Characteristics of gut microbiota in adult patients with type 1 and type 2 diabetes based on next-generation sequencing of the $16 \mathrm{~S}$ rRNA gene fragment," Polish Archives of Internal Medicine, vol. 128, no. 6, pp. 336-343, 2018.

[26] P. F. de Groot, C. Belzer, Ö. Aydin et al., "Distinct fecal and oral microbiota composition in human type 1 diabetes, an observational study," PLoS One, vol. 12, no. 12, article e0188475, 2017.

[27] S. Pellegrini, V. Sordi, A. M. Bolla et al., "Duodenal mucosa of patients with type 1 diabetes shows distinctive inflammatory profile and microbiota," The Journal of Clinical Endocrinology and Metabolism, vol. 102, no. 5, pp. 1468-1477, 2017.

[28] C. J. Stewart, A. Nelson, M. D. Campbell et al., "Gut microbiota of type 1 diabetes patients with good glycaemic control and high physical fitness is similar to people without diabetes: an observational study," Diabetic Medicine, vol. 34, no. 1, pp. 127-134, 2017.

[29] C. J. Qi, Q. Zhang, M. Yu et al., "Imbalance of fecal microbiota at newly diagnosed type 1 diabetes in Chinese children," Chinese Medical Journal, vol. 129, no. 11, pp. 1298-1304, 2016.

[30] M. E. Mejía-León, J. F. Petrosino, N. J. Ajami, M. G. Domínguez-Bello, and A. M. C. de la Barca, "Fecal microbiota imbalance in Mexican children with type 1 diabetes," Scientific Reports, vol. 4, no. 1, article 3814, 2015.

[31] A. Giongo, K. A. Gano, D. B. Crabb et al., "Toward defining the autoimmune microbiome for type 1 diabetes," ISME Journal, vol. 5, no. 1, pp. 82-91, 2011.

[32] C. T. Brown, A. G. Davis-Richardson, A. Giongo et al., "Gut microbiome metagenomics analysis suggests a functional model for the development of autoimmunity for type 1 diabetes," PLoS One, vol. 6, no. 10, article e25792, 2011.

[33] M. C. de Goffau, K. Luopajarvi, M. Knip et al., "Fecal microbiota composition differs between children with $\beta$-cell autoimmunity and those without," Diabetes, vol. 62, no. 4, pp. 1238-1244, 2013.

[34] A. D. Kostic, D. Gevers, H. Siljander et al., "The dynamics of the human infant gut microbiome in development and in progression toward type 1 diabetes," Cell Host \& Microbe, vol. 17, no. 2, pp. 260-273, 2015.

[35] T. R. Abrahamsson, H. E. Jakobsson, A. F. Andersson, B. Björkstén, L. Engstrand, and M. C. Jenmalm, "Low diversity of the gut microbiota in infants with atopic eczema," Journal of Allergy and Clinical Immunology, vol. 129, no. 2, pp. 434440.e2, 2012.

[36] E. Papa, M. Docktor, C. Smillie et al., "Non-invasive mapping of the gastrointestinal microbiota identifies children with inflammatory bowel disease," PLoS One, vol. 7, no. 6, article e39242, 2012.

[37] T. Lu, Y. Chen, Y. Guo et al., "Altered gut microbiota diversity and composition in chronic urticaria," Disease Markers, vol. 2019, Article ID 6417471, 11 pages, 2019.

[38] S. L. Russell, M. J. Gold, M. Hartmann et al., "Early life antibioticdriven changes in microbiota enhance susceptibility to allergic asthma," EMBO Reports, vol. 13, no. 5, pp. 440-447, 2012. 
[39] D. Gong, X. Gong, L. Wang, X. Yu, and Q. Dong, "Involvement of reduced microbial diversity in inflammatory bowel disease," Gastroenterology Research and Practice, vol. 2016, Article ID 6951091, 7 pages, 2016.

[40] D. R. Ownby, C. C. Johnson, and E. L. Peterson, "Exposure to dogs and cats in the first year of life and risk of allergic sensitization at 6 to 7 years of age," JAMA, vol. 288, no. 8, pp. 963-972, 2002.

[41] G. A. W. Rook and J. L. Stanford, "Give us this day our daily germs," Immunology Today, vol. 19, no. 3, pp. 113-116, 1998.

[42] M. Murri, I. Leiva, J. M. Gomez-Zumaquero et al., "Gut microbiota in children with type 1 diabetes differs from that in healthy children: a case-control study," BMC Medicine, vol. 11, no. 1, 2013.

[43] R. S. Longman, Y. Yang, G. E. Diehl, S. V. Kim, and D. R. Littman, "Microbiota: host interactions in mucosal homeostasis and systemic autoimmunity," Cold Spring Harbor Symposia on Quantitative Biology, vol. 78, pp. 193-201, 2014.

[44] M. Rescigno, "Intestinal microbiota and its effects on the immune system," Cellular Microbiology, vol. 16, no. 7, pp. 1004-1013, 2014.

[45] A. Tanca, A. Palomba, C. Fraumene, V. Manghina, M. Silverman, and S. Uzzau, "Clostridial butyrate biosynthesis enzymes are significantly depleted in the gut microbiota of nonobese diabetic mice," mSphere, vol. 3, no. 5, 2018.

[46] K. Atarashi, T. Tanoue, T. Shima et al., "Induction of colonic regulatory T cells by indigenous Clostridium species," Science, vol. 331, no. 6015, pp. 337-341, 2011.

[47] L. Peng, Z. R. Li, R. S. Green, I. R. Holzman, and J. Lin, "Butyrate enhances the intestinal barrier by facilitating tight junction assembly via activation of AMP-activated protein kinase in Caco-2 cell monolayers," The Journal of Nutrition, vol. 139, no. 9, pp. 1619-1625, 2009.

[48] A. Hague, A. J. Butt, and C. Paraskeva, "The role of butyrate in human colonic epithelial cells: an energy source or inducer of differentiation and apoptosis?," The Proceedings of the Nutrition Society, vol. 55, no. 3, pp. 937-943, 1996.

[49] T. Watts, I. Berti, A. Sapone et al., "Role of the intestinal tight junction modulator zonulin in the pathogenesis of type I diabetes in BB diabetic-prone rats," Proceedings of the National Academy of Sciences of the United States of America, vol. 102, no. 8, pp. 2916-2921, 2005.

[50] A. Tarko, A. Suchojad, M. Michalec, M. Majcherczyk, A. Brzozowska, and I. Maruniak-Chudek, "Zonulin: a potential marker of intestine injury in newborns," Disease Markers, vol. 2017, Article ID 2413437, 6 pages, 2017.

[51] C. V. Ferreira-Halder, A. V. . S. Faria, and S. S. Andrade, "Action and function of Faecalibacterium prausnitzii in health and disease," Best Practice \& Research. Clinical Gastroenterology, vol. 31, no. 6, pp. 643-648, 2017.

[52] D. Viggiano, G. Ianiro, G. Vanella et al., "Gut barrier in health and disease: focus on childhood," European Review for Medical and Pharmacological Sciences, vol. 19, no. 6, pp. 1077-1085, 2015.

[53] K. Vehik and D. Dabelea, "The changing epidemiology of type 1 diabetes: why is it going through the roof?," Diabetes/Metabolism Research and Reviews, vol. 27, no. 1, pp. 3-13, 2011.

[54] N. Burger-van Paassen, A. Vincent, P. J. Puiman et al., "The regulation of intestinal mucin MUC2 expression by shortchain fatty acids: implications for epithelial protection," The Biochemical Journal, vol. 420, no. 2, pp. 211-219, 2009.
[55] H. J. Flint, K. P. Scott, S. H. Duncan, P. Louis, and E. Forano, "Microbial degradation of complex carbohydrates in the gut," Gut Microbes, vol. 3, no. 4, pp. 289-306, 2014.

[56] H. Tlaskalová-Hogenová, R. Štěpánková, H. Kozáková et al., "The role of gut microbiota (commensal bacteria) and the mucosal barrier in the pathogenesis of inflammatory and autoimmune diseases and cancer: contribution of germ-free and gnotobiotic animal models of human diseases," Cellular \& Molecular Immunology, vol. 8, no. 2, pp. 110-120, 2011.

[57] T. Vatanen, A. D. Kostic, E. d'Hennezel et al., "Variation in microbiome LPS immunogenicity contributes to autoimmunity in humans," Cell, vol. 165, no. 4, pp. 842-853, 2016.

[58] M. Rajilić-Stojanović, D. M. Jonkers, A. Salonen et al., "Intestinal microbiota and diet in IBS: causes, consequences, or epiphenomena?," The American Journal of Gastroenterology, vol. 110, no. 2, pp. 278-287, 2015.

[59] A. O'Callaghan and D. van Sinderen, "Bifidobacteria and their role as members of the human gut microbiota," Frontiers in Microbiology, vol. 7, p. 925, 2016.

[60] S. H. Duncan, P. Louis, and H. J. Flint, "Lactate-utilizing bacteria, isolated from human feces, that produce butyrate as a major fermentation product," Applied and Environmental Microbiology, vol. 70, no. 10, pp. 5810-5817, 2004.

[61] M. A. Underwood, J. B. German, C. B. Lebrilla, and D. A. Mills, "Bifidobacterium longum subspecies infantis: champion colonizer of the infant gut," Pediatric Research, vol. 77, no. 1-2, pp. 229-235, 2015.

[62] C. O'Mahony, P. Scully, D. O'Mahony et al., "Commensalinduced regulatory $\mathrm{T}$ cells mediate protection against pathogen-stimulated NF-kappaB activation," PLoS Pathogens, vol. 4, no. 8, article e1000112, 2008.

[63] S. G. Jeon, H. Kayama, Y. Ueda et al., "Probiotic Bifidobacterium breve induces IL-10-producing Tr1 cells in the colon," PLoS Pathogens, vol. 8, no. 5, article e1002714, 2012.

[64] I. Cosorich, G. Dalla-Costa, C. Sorini et al., "High frequency of intestinal TH17 cells correlates with microbiota alterations and disease activity in multiple sclerosis," Science Advances, vol. 3, no. 7, article e1700492, 2017.

[65] M. Jahromi and E. Al-Ozairi, "Human leukocyte antigen (HLA) and islet autoantibodies are tools to characterize type 1 diabetes in Arab countries: emphasis on Kuwait," Disease Markers, vol. 2019, Article ID 9786078, 10 pages, 2019.

[66] L. Jia, K. Shan, L. L. Pan et al., "Clostridium butyricum CGMCC0313.1 protects against autoimmune diabetes by modulating intestinal immune homeostasis and inducing pancreatic regulatory T cells," Frontiers in Immunology, vol. 8, article 1345, 2017.

[67] F. Calcinaro, S. Dionisi, M. Marinaro et al., "Oral probiotic administration induces interleukin-10 production and prevents spontaneous autoimmune diabetes in the non-obese diabetic mouse," Diabetologia, vol. 48, no. 8, pp. 15651575, 2005.

[68] E. Mariño, J. L. Richards, K. H. McLeod et al., "Gut microbial metabolites limit the frequency of autoimmune $\mathrm{T}$ cells and protect against type 1 diabetes," Nature Immunology, vol. 18, no. 5, pp. 552-562, 2017.

[69] J. Ho, A. C. Nicolucci, H. Virtanen et al., "Effect of prebiotic on microbiota, intestinal permeability and glycemic control in children with type 1 diabetes," The Journal of Clinical Endocrinology and Metabolism, vol. 104, no. 10, pp. 4427-4440, 2019. 
[70] E. G. Zoetendal, A. von Wright, T. Vilpponen-Salmela, K. BenAmor, A. D. L. Akkermans, and W. M. de Vos, "Mucosa-associated bacteria in the human gastrointestinal tract are uniformly distributed along the colon and differ from the community recovered from feces," Applied and Environmental Microbiology, vol. 68, no. 7, pp. 3401-3407, 2002. 